\title{
Interactive comment on "Acoustic wave propagation in rivers: an experimental study" by Thomas Geay et al.
}

\section{Anonymous Referee \#1}

Received and published: 25 January 2019

Review of 'Acoustic wave propagation in rivers: an experimental study' by Geay et al

\section{General comment.}

The manuscript reports on underwater measurements of ambient acoustic noise levels collected in several shallow rivers in the French Alps. The rational for collecting the data is to improve the measurement of the bedload gravel transport, using passive underwater acoustic receivers, hydrophones. Unfortunately, there was an order of magnitude variation in the acoustical noise levels between the different rivers. This variability complicates the generic application of the data to the enhancement of passive acoustic detection of gravel transport. However, given the limited studies of ambient acoustical noise levels in rivers, the data does provide indicative background sound levels, which may be of some value for the passive acoustic detection of gravel transport. 
The study may also be of interest to others concerned with acoustical riverine noise levels e.g. naval, marine noise pollution etc. The publication of the work could possibly be considered to be of broader interest than solely the gravel transport community.

\section{Specific comments}

1 In the abstract the word 'rugosity' is used, this word is not in common usage; the selection of an alternative to describe this feature of the bed would be helpful.

2 P2 line 5 'frequential characteristics' is a slightly odd phrase, 'spectral characteristics' would be more commonly used.

3 P3 line 11 'interfaces are totally transparent, acoustic waves propagate' it is unlikely that the interfaces would be 'totally transparent', however, they could be 'highly absorbing'.

4 P3 line 20 ' $c$ is the celerity of the acoustic waves in water ( $\mathrm{m} / \mathrm{s})$ '; why not simply say ' $c$ is the velocity of sound in water $(\mathrm{m} / \mathrm{s})$ '?

5 On P4 and in figure 2 the transmit sensitivity of the underwater loudspeaker is presented, however, this is only valid if the hydrophones have uniform receive sensitivity over the bandwidth of the transmitter. What was the receiver response over the transmit bandwidth?

6 P4 line 29 It is not clear what is meant by 'shared' in 'The system is shared by a Carlson river board'. Was it 'mounted' on a Carlson river board?

$7 \mathrm{P} 4$ line 30/31 'Lagrangian measurements were preferred to fix-position measurements to optimize the signal to noise ratio.' A few words explaining why this was 'optimize' would be useful.

8 P5 line 12 'describes how are processed the hydrophone signals' 'how the hydrophone signals were processed' would be better.

9 P7 line 5 and fig 5. Some explanation needs to be provided for choosing $1.0 \mathrm{kHz}$ to 
assess the acoustic power with range, given that it is cited on $\mathrm{P} 7$ line 16 'that estimate a cut-off frequency around1.1 kHz' Why choose to use $1.0 \mathrm{kHz}$ when it is below the ESurfD cut-off frequency?

10 P7 line 9 'is repeated' should be 'was repeated'.

$11 \mathrm{P} 7 \mathrm{lt}$ is not clear in the text how figures $6 \mathrm{a}$ and $6 \mathrm{~b}$ were obtained from the data and how they relate to figure 5 . Given this process is central to the manuscript output, it needs to be explicitly and clearly explained. Are the measured spectra in the rivers being scaled to the lake spectra at $1.0 \mathrm{kHz}$ ? Are the lake spectral levels being used to obtain the attenuation? Are spectral measurements at different ranges used to calculate the riverine attenuation? Clarification is required if the manuscript is to be published.

$12 \mathrm{P} 7$ As with point 11 above it is not clear how the spectra in figure 7 and attenuations in figure 8 were actually obtained from the measurements. Again further clarification is required if the manuscript is to be published. It is not possible to ascertain the veracity of the results presented due to a lack of a clear explanation of the data analysis process.

13 P9 line 2 There needs to be some justification for the choice of $1600 \mathrm{~m} / \mathrm{s}$ for the sound velocity in the bed sediments.

14 P9 line 9 'The variation of attenuation coefficients at higher frequencies is here discussed' It would be useful to compare the measured attenuations with that calculated solely by the absorption due to the water itself. Was the water absorption a significant component of the measured attenuation in any of the rivers?

15 P10 equation 10. It may be interesting to present equation 10. However, how would the attenuation coefficient be obtained for a new river in which SGN PSD measurements were being collected?

Printer-friendly version

$16 \mathrm{P} 11$ line 16 ' $\alpha \lambda$ is higher for higher bed slopes of the river'. Any physical explanation 


\section{for this?}

The manuscript presents a series of observations, which require further explanation ESurfD as to how the attenuation and source levels are obtained over the spectra presented. In addition, because no ancillary data were collected on the sediments beds and water surface roughness the results presented are of limited value. However, there are not many measurements of riverine soundscapes and therefore it could be considered a publishable manuscript if this is deemed sufficiently original.

Please also note the supplement to this comment:

https://www.earth-surf-dynam-discuss.net/esurf-2018-80/esurf-2018-80-RC1supplement.pdf

Interactive comment on Earth Surf. Dynam. Discuss., https://doi.org/10.5194/esurf-2018-80, 2018. 